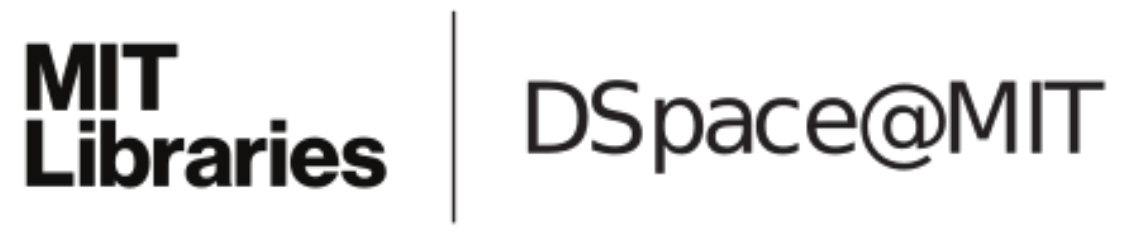

\author{
MIT Open Access Articles
}

Direct-gap optical gain of Ge on Si at room temperature

The MIT Faculty has made this article openly available. Please share how this access benefits you. Your story matters.

Citation: Liu, Jifeng, Xiaochen Sun, Lionel C. Kimerling, and Jurgen Michel. “Direct-gap optical gain of Ge on Si at room temperature." Optics Letters 34, no. 11 (May 29, 2009): 1738. ( 2009 Optical Society of America.

As Published: http://dx.doi.org/10.1364/OL.34.001738

Publisher: Optical Society of America

Persistent URL: http://hdl.handle.net/1721.1/81205

Version: Final published version: final published article, as it appeared in a journal, conference proceedings, or other formally published context

Terms of Use: Article is made available in accordance with the publisher's policy and may be subject to US copyright law. Please refer to the publisher's site for terms of use. 


\title{
Direct-gap optical gain of Ge on Si at room temperature
}

\author{
Jifeng Liu,* Xiaochen Sun, Lionel C. Kimerling, and Jurgen Michel \\ Department of Materials Science and Engineering, Microphotonics Center, Massachusetts Institute of Technology, \\ Cambridge, Massachusetts 02139, USA \\ *Corresponding author: jfliu01@mit.edu
}

Received March 13, 2009; revised April 23, 2009; accepted April 26, 2009; posted April 30, 2009 (Doc. ID 108596); published May 29, 2009

Lasers on Si are crucial components of monolithic electronic-photonic integration. Recently our theoretical analysis has shown that Ge, a pseudodirect bandgap material compatible with Si complementary metal oxide semiconductor technology, can be band engineered by tensile strain and $n$-type doping to achieve efficient light emission and optical gain from its direct gap transition. We report on what is to our knowledge the first experimental observation of optical gain in the wavelength range of 1600-1608 nm from the direct-gap transition of $n^{+}$tensile-strained Ge on $\mathrm{Si}$ at room temperature under steady-state optical pumping. This experimental result confirms that the band-engineered $\mathrm{Ge}$ on $\mathrm{Si}$ is a promising gain medium for monolithic lasers on Si. (C) 2009 Optical Society of America

OCIS codes: $130.5990,160.3380,250.5980$.

High-performance electrically pumped light emitters on silicon have long been one of the most crucial devices yet to be achieved by monolithic electronicphotonic integration [1]. Investigations in this field include porous Si [2], Si nanocrystals [3], Er-doped Si or Si nanostructures [4,5], SiGe nanostructures [6], GeSn [7], $\beta-\mathrm{FeSi}_{2}$ [8], and hybrid III-V lasers on $\mathrm{Si}$ $[9,10]$. Germanium-based materials on $\mathrm{Si}$ are particularly interesting for this application, since they have already been widely applied to advanced electronic devices such as strain-enhanced high mobility complementary metal oxide semiconductor (CMOS) transistors [11], as well as integrated photonic devices such as waveguide-coupled photodetectors and electroabsorption modulators that are CMOS compatible $[12,13]$. If a high-performance Ge-on-Si light source exists, all active photonic devices on Si can be realized using Ge, which greatly simplifies the monolithic electronic-photonic integration process. Germanium is a pseudodirect gap material, since the energy difference between its direct $(\Gamma)$ and indirect $(L)$ bandgaps is only $136 \mathrm{meV}$ [14]. Recently, our theoretical analysis has indicated that Ge can be band engineered by tensile strain and $n$-type doping to achieve efficient light emission and optical gain from its direct gap transition within the third optical communication window (approximately 1520-1620 nm) [15]. Indeed, direct-gap photoluminescence (PL) [16,17] and electroluminescence (EL) [18] at room temperature have already been demonstrated from these band-engineered Ge-on-Si materials. In this Letter, we report the first (to our knowledge) experimental observation of optical gain in the wavelength range of 1600-1608 nm from the direct gap transition of $n^{+}$ tensile-strained $\mathrm{Ge}$ on $\mathrm{Si}$ at room temperature under a steady-state optical pumping of $\sim 7.0 \mathrm{~kW} / \mathrm{cm}^{2}$. An optical gain coefficient of $\sim 50 \mathrm{~cm}^{-1}$ was achieved at $1605 \mathrm{~nm}$. These experimental results confirm that the band-engineered $\mathrm{Ge}$ on $\mathrm{Si}$ is a promising gain medium for monolithic lasers on Si.

Ge mesas with in situ phosphorous doping were se- lectively grown on $\mathrm{Si}$ by ultrahigh vacuum chemical vapor deposition (UHVCVD) using an $\mathrm{SiO}_{2}$ mask layer. The details about this two-step growth method were reported earlier [19]. The samples were annealed by rapid thermal annealing at $780^{\circ} \mathrm{C}$ for $30 \mathrm{~s}$ to reduce the defect density [19]. The Ge layer was nearly fully relaxed at the growth and annealing temperatures, and tensile strain was accumulated upon cooling to room temperature owing to the large thermal expansion coefficient difference between Ge and Si $[20,21]$. The thermally induced tensile strain in the Ge layer is $0.23 \%$ and the corresponding direct bandgaps from the maxima of light $(l h)$ and heavy $(h h)$ hole bands to the minimum of the $\Gamma$ valley are $E_{g}^{\Gamma}(l h)=0.767 \mathrm{eV}$ and $E_{g}^{\Gamma}(h h)=0.782 \mathrm{eV}$, respectively [21].

To investigate the optical bleaching and the gain of $n^{+}$tensile-strained Ge on $\mathrm{Si}$, a pump-probe measurement was performed using the setup schematically shown in the inset of Fig. 1. A $1480 \mathrm{~nm} \mathrm{cw}$ pump laser and a tunable probe laser with an output wavelength range of 1510-1640 $\mathrm{nm}$ were coupled into a single-mode lens-tipped optical fiber through a wavelength division multiplexing (WDM) coupler. The light was incident on the front surface of the sample. A selectively grown $n^{+}$Ge mesa with an area of $500 \mu \mathrm{m}^{2}$ and an active doping level of $n=1.0$ $\times 10^{19} \mathrm{~cm}^{-3}$ was used in this study. The $\mathrm{SiO}_{2}$ mask layer for the selective growth naturally provides carrier confinement in the lateral direction to enhance the injected carrier concentration in the selectively grown Ge mesa. The Ge film grown on the backside of the Si wafer during the UHVCVD process was removed to simplify data analysis. The Ge layer on the front side was $870 \mathrm{~nm}$ thick. An InGaAs detector with an integration sphere was placed at the backside of the sample to collect the transmitted signal. To accurately measure the transmittance of the probe laser through the $n^{+}$Ge-on-Si mesa, the probe laser was modulated at $500 \mathrm{~Hz}$ internally and a lock-in approach was adopted to record the transmitted probe 


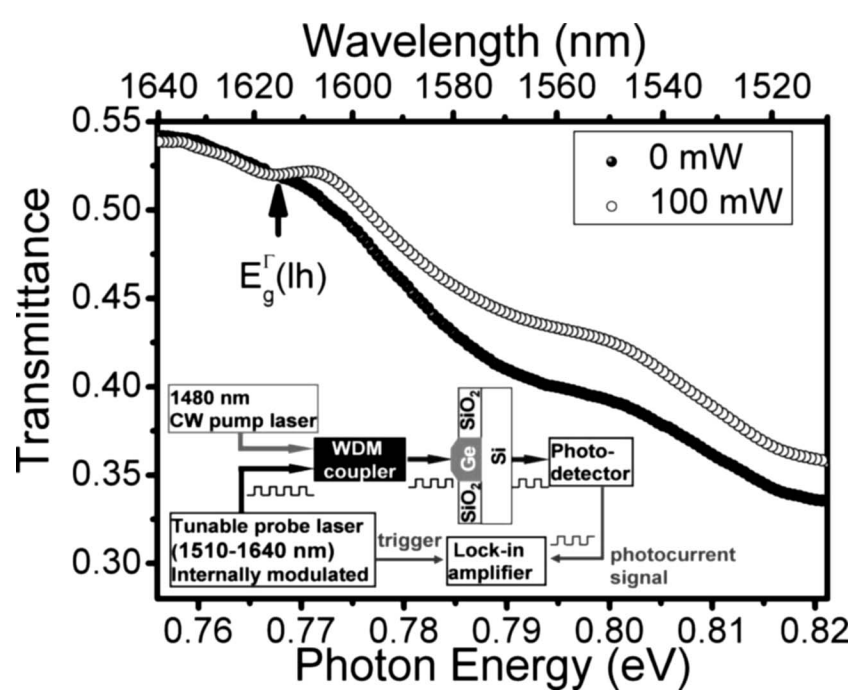

Fig. 1. Transmittance spectra of a $500 \mu \mathrm{m}^{2}$ Ge-on-Si mesa sample with $n=1.0 \times 10^{19} \mathrm{~cm}^{-3}$ under 0 and $100 \mathrm{~mW}$ optical pumping. The inset shows a schematic of the pump-probe measurement setup.

laser signal only. This method achieves a $\pm 0.25 \%$ accuracy in the transmittance measurement of the probe laser, and to be more conservative we adopted an accuracy of $\pm 0.4 \%$ to reflect the upper limit of the transmittance measurement error in the data analysis. Compared with the commonly used pulse laser pumping in pump-probe measurements that emphasizes transient behavior, the optical bleaching measured under cw pumping in this case reflects the properties of the band-engineered Ge-on-Si sample at a steady-state injection level, which is more relevant to the real operation of laser devices.

The transmittance spectra of the sample under 0 and $100 \mathrm{~mW}$ optical pumping are shown in Fig. 1. The effective pump power density at $100 \mathrm{~mW}$ is estimated to be $\sim 7.0 \mathrm{~kW} / \mathrm{cm}^{2}$ considering coupling loss at the fiber connectors and the effective absorption of the pump laser by the thin Ge layer. Without optical pumping, the transmittance starts to decrease dramatically at photon energies $>0.767 \mathrm{eV}$ owing to the onset of the direct gap absorption $\left[E_{g}^{\Gamma}(l h)=0.767 \mathrm{eV}\right]$. Upon optical pumping, we observe that the transmittance at photon energies $>0.767 \mathrm{eV}$ significantly increases while the transmittance at photon energies $<0.767 \mathrm{eV}$ slightly decreases. These experimental results indicate that the bleaching of the direct gap absorption dominates the increase in the free-carrier absorption at photon energies above the direct bandgap upon optical pumping, while below the direct bandgap the increase in the free-carrier absorption dominates the change in transmittance.

To derive the absorption spectra of the sample under 0 and $100 \mathrm{~mW}$ optical pumping from the transmittance data in Fig. 1, we use the transfer matrix method [22] and the Kramers-Kronig relation to solve both the real refractive index $\left(n_{r}\right)$ and the absorption coefficient $(\alpha)$ deterministically using an iterative self-consistent regression approach. We substitute the refractive index $n_{r}(\lambda)$ of bulk Ge [14] into the transfer matrix equation as a starting point to solve $\alpha(\lambda)$ from the transmittance data, then we use the newly derived $\alpha(\lambda)$ to obtain a corrected $n_{r}(\lambda)$ through the Kramers-Kronig relation. This process is iterated back and forth until self-consistency is obtained for both the transfer matrix equation of transmittance and the Kramers-Kronig equation. As an example, the derived absorption spectrum and refractive index spectrum of the tensile-strained $n^{+} \mathrm{Ge}$ mesa on Si without optical pumping are compared with those of bulk Ge in Fig. 2. The absorption edge is significantly redshifted owing to the bandgap shrinkage induced by the tensile strain $[20,21]$. As a result, the refractive index is also notably modified compared with bulk Ge owing to the KramersKronig relation. Such a self-consistency approach guarantees a more accurate solution to the absorption coefficients than simply assuming bulk Ge refractive index for tensile-strained Ge in the transfer matrix calculation. In fact, the absorption spectrum derived here is in good agreement with a recent report on the absorption of tensile-strained Ge-on-Si films [23].

Figure 3 shows the derived absorption spectra of the $n^{+}$Ge mesa sample under 0 and $100 \mathrm{~mW}$ optical pumping. The absorption coefficients at photon energies of $>0.767 \mathrm{eV} \quad(\lambda<1617 \mathrm{~nm})$ decrease significantly upon optical pumping. In particular, negative absorption coefficients corresponding to optical gain are observed in the wavelength range of 1600-1608 $\mathrm{nm}$, as shown in the inset of Fig. 3. The shape of the gain spectrum near the direct band edge of Ge resembles those of III-V semiconductor materials [22]. The maximum gain coefficient observed is $\gamma=-\alpha$ $=56 \pm 25 \mathrm{~cm}^{-1}$ at $1605 \mathrm{~nm}$. The error bars given here reflect the upper limit of the transmittance measurement error of $\pm 0.4 \%$. Even with the most conservative estimate, a gain coefficient of $>25 \mathrm{~cm}^{-1}$ at 1605 $\mathrm{nm}$, which is equivalent to $>100 \mathrm{~dB} \mathrm{~cm}^{-1}$, is guaranteed. This gain coefficient is much greater than the waveguide loss in Si photonics (typically $<10 \mathrm{~dB}$ $\mathrm{cm}^{-1}$ ) and can be applied to optical gain devices on chip. To our knowledge, this is the first time that optical gain has been observed from the direct-gap

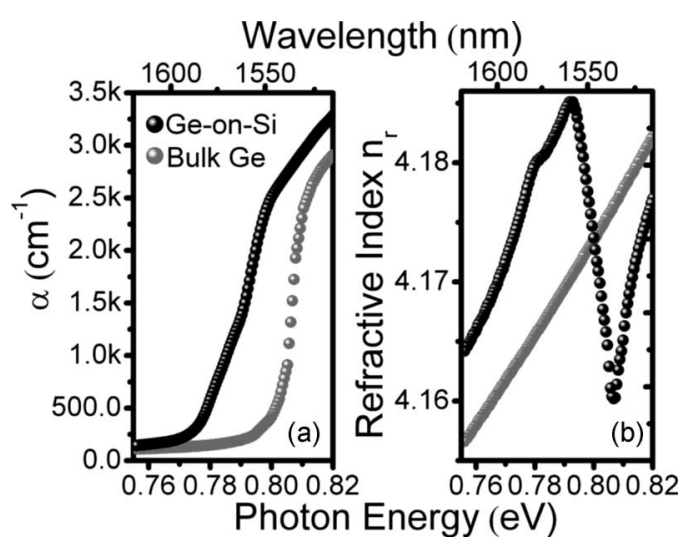

Fig. 2. Comparison of (a) absorption spectra and (b) refractive index spectra of bulk Ge with the tensile-strained $n^{+}$Ge-on-Si mesa sample. 


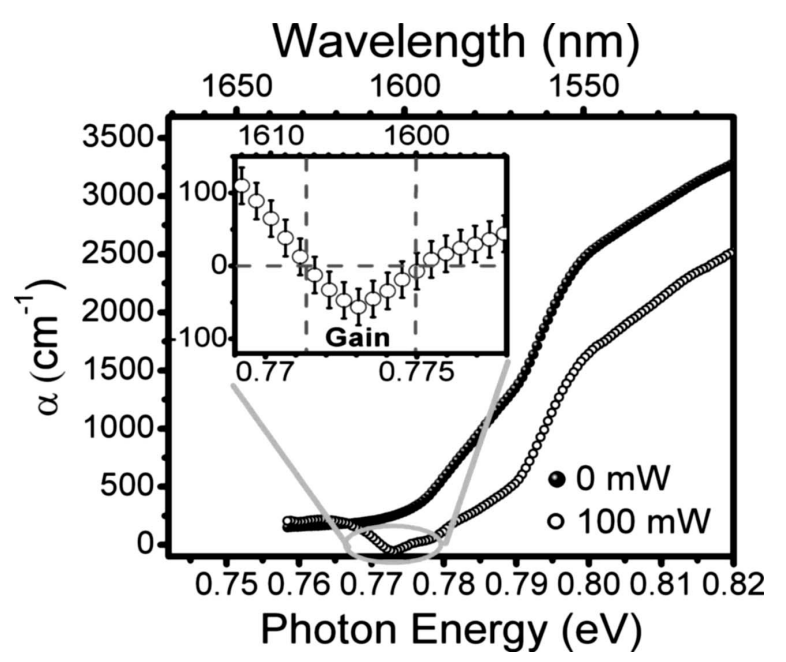

Fig. 3. Absorption spectra of the $n^{+}$Ge mesa sample under 0 and $100 \mathrm{~mW}$ optical pumping. Negative absorption coefficients corresponding to optical gain are observed in the wavelength range of 1600-1608 nm, as shown in the inset. The error bars reflect the upper limit of the transmittance measurement error of $\pm 0.4 \%$.

transition of Ge. This result indicates that bandengineered Ge can be applied as an optical gain medium for monolithically integrated lasers and optical amplifiers on Si. Further improvement in gain coefficients is expected with a higher $n$-type doping level and is supported by the trend in PL intensity enhancement $[16,17]$. Increasing $n$-type doping in Ge is also expected to extend optical gain to the $C$ band $(1528-1560 \mathrm{~nm})$ at $>5 \times 10^{18} \mathrm{~cm}^{-3}$ injection levels [15].

In conclusion, we report the first observation of optical gain in epitaxial $\mathrm{Ge}$ on $\mathrm{Si}$ at room temperature by using tensile strain and $n$-type doping for band engineering. The optical gain has been observed in the wavelength range of 1600-1608 $\mathrm{nm}$ near the direct bandgap of the band-engineered Ge mesa on Si and a maximum gain coefficient of $\gamma=56 \pm 25 \mathrm{~cm}^{-1}$ was observed at $1605 \mathrm{~nm}$. This gain coefficient is much greater than the waveguide loss in Si photonics even with the most conservative estimate and can be applied to optical gain devices on chip. These results demonstrate the potential of band-engineered Ge as an optical gain medium for monolithically integrated lasers on Si.

This work was supported by the Si-based Laser Initiative of the Multidisciplinary University Research Initiative (MURI) sponsored by the Air Force Office of Scientific Research (AFOSR) and supervised by Gernot Pomrenke.

\section{References}

1. D. J. Lockwood and L. Pavesi, Silicon Photonics (Springer-Verlag, 2004), p. 50.

2. N. Koshida and H. Koyama, Appl. Phys. Lett. 60, 347 (1992).

3. L. Pavesi, L. Dal Negro, C. Mazzoleni, G. Franzo, and F. Priolo, Nature 408, 440 (2000).

4. B. Zheng, J. Michel, F. Y. G. Ren, L. C. Kimerling, D. C. Jacobson, and J. M. Poate, Appl. Phys. Lett. 64, 2842 (1994).

5. A. J. Kenyon, P. F. Trwoga, M. Federighi, and C. W. Pitt, J. Phys.: Condens. Matter 6, L319 (1994).

6. C. S. Peng, Q. Huang, W. Q. Cheng, J. M. Zhou, Y. H. Zhang, T. T. Sheng, and C. H. Tung, Phys. Rev. B 57, 8805 (1998).

7. G. He and H. A. Atwater, Phys. Rev. Lett. 79, 1937 (1997).

8. D. Leong, J. Harry, K. J. Reeson, and K. P. Homewood, Nature 387, 686 (1997).

9. M. E. Groenert, C. W. Leitz, A. J. Pitera, V. Yang, H. Lee, R. J. Ram, and E. A. Fitzgerald, J. Appl. Phys. 93, 362 (2003).

10. A. W. Fang, H. Park, O. Cohen, R. Jones, M. J. Paniccia, and J. E. Bowers, Opt. Express 14, 9203 (2006).

11. M. J. Lee and E. A. Fitzgerald, J. Appl. Phys. 97, 011101 (2005).

12. D. H. Ahn, C. Y. Hong, J. F. Liu, M. Beals, W. Giziewicz, L. C. Kimerling, and J. Michel, Opt. Express 15, 3916 (2007).

13. J. F. Liu, M. Beals, A. Pomerene, S. Bernardis, R. Sun, J. Cheng, L. C. Kimerling, and J. Michel, Nat. Photonics 2, 433 (2008).

14. Physics of Group IV Elements and III-V Compounds, Vol. 17a of Landolt-Börnstein: Numerical Data and Functional Relationships in Science and Technology, O. Madelung, ed. (Springer, 1982).

15. J. Liu, X. Sun, D. Pan, X. X. Wang, L. C. Kimerling, T. L. Koch, and J. Michel, Opt. Express 15, 11272 (2007).

16. J. F. Liu, X. C. Sun, P. Becla, L. C. Kimerling, and J. Michel, The Fifth IEEE International Conference on Group IV Photonic (2008), pp. 16-18.

17. X. C. Sun, J. F. Liu, L. C. Kimerling, J. Michel, and T. L. Koch, 2008 OSA Conference on Integrated Photonics and Nanophotonics Research and Application (Optical Society of America, 2008), paper IMC5.

18. X. C. Sun, J. F. Liu, L. C. Kimerling, and J. Michel, Opt. Lett. 34, 1198 (2009).

19. H. Luan, D. R. Lim, K. K. Lee, K. M. Chen, J. G. Sandland, K. Wada, and L. C. Kimerling, Appl. Phys. Lett. 75, 2909 (1999).

20. Y. Ishikawa, K. Wada, D. D. Cannon, J. F. Liu, H. C. Luan, and L. C. Kimerling, Appl. Phys. Lett. 82, 2044 (2003).

21. J. F. Liu, D. D. Cannon, K. Wada, Y. Ishikawa, D. T. Danielson, S. Jongthammanurak, J. Michel, and L. C. Kimerling, Phys. Rev. B 70, 155309 (2004).

22. S. L. Chuang, Physics of Optoelectronic Devices (Wiley, 1995).

23. V. Sorianello, A. Perna, L. Colace, G. Assanto, H. C. Luan, and L. C. Kimerling, Appl. Phys. Lett. 93, 111115 (2008). 